\title{
A high-throughput fluorescence polarization assay for discovering inhibitors targeting the DNA-binding domain of signal transducer and activator of transcription 3 (STAT3)
}

\author{
Po-Chang Shih ${ }^{1}$, Yiwen Yang ${ }^{1}$, Gary N. Parkinson ${ }^{1}$, Andrew Wilderspin ${ }^{1}$ and \\ Geoffrey Wells ${ }^{1}$ \\ ${ }^{1}$ UCL School of Pharmacy, University College London, Bloomsbury, London WC1N 1AX, UK \\ Correspondence to: Geoffrey Wells, email: g.wells@ucl.ac.uk \\ Keywords: STAT3; signal transducer and activator of transcription; high-throughput; DNA-binding domain; fluorescence polarization \\ Received: March 27, $2018 \quad$ Accepted: July 31, $2018 \quad$ Published: August 24, 2018 \\ Copyright: Shih et al. This is an open-access article distributed under the terms of the Creative Commons Attribution License 3.0 \\ (CC BY 3.0), which permits unrestricted use, distribution, and reproduction in any medium, provided the original author and source \\ are credited.
}

\section{ABSTRACT}

Anti-cancer drug discovery efforts to directly inhibit the signal transducer and activator of transcription 3 (STAT3) have been active for over a decade following the discovery that $70 \%$ of cancers exhibit elevated STAT3 activity. The majority of research has focused on attenuating STAT3 activity through preventing homo-dimerization by targeting the SH2 or transcriptional activation domains. Such dimerization inhibitors have not yet reached the market. However, an alternative strategy focussed on preventing STAT3 DNA-binding through targeting the DNA-binding domain (DBD) offers new drug design opportunities. Currently, only EMSA and ELISA-based methods have been implemented with suitable reliability to characterize STAT3 DBD inhibitors. Herein, we present a new orthogonal, fluorescence polarization (FP) assay suitable for high-throughput screening of molecules. This assay, using a STAT3 ${ }^{127-688}$ construct, was developed and optimized to screen molecules that attenuate the STAT3:DNA association with good reliability $\left(Z^{\prime}\right.$ value $>0.6$ ) and a significant contrast (signal-tonoise ratio $>15.0$ ) at equilibrium. The assay system was stable over a 48 hour period. Significantly, the assay is homogeneous and simple to implement for high-throughput screening compared to EMSA and ELISA. Overall, this FP assay offers a new way to identify and characterize novel molecules that inhibit STAT3:DNA association.

\section{INTRODUCTION}

STAT3, signal transducer and activator of transcription 3 , is a key component in several signalling pathways $[1,2]$, is over-activated in approximately $70 \%$ of cancers [3] and plays critical roles in cell proliferation, cell survival, angiogenesis, immune invasion and metastasis [4]. Intensive efforts have been made over more than a decade to discover and develop small-molecule inhibitors to abate STAT3 activity through targeting its $\mathrm{SH} 2$ or transcriptional activation domains for the lowering of dimerization and activation of the protein [5-17]. During the discovery of STAT3 dimerization inhibitors, numerous cell-free and cell-based assays were developed to validate the inhibitory effect of selective inhibitors on dimerization. These include assays based on highthroughput fluorescence polarization (FP) [10, 13, 15, 18], AlphaScreen $^{\text {TM }}[6,7,19]$, fluorescence resonance energy transfer (FRET) [6, 9, 20], enzyme-linked immunosorbent assays (ELISA) [21], cytoblot [5, 22, 23], and surface plasmon resonance (SPR) methodologies [8]. The FP, AlphaScreen ${ }^{\mathrm{TM}}$, ELISA and SPR assays are all applicable to high-throughput screening and are cell-free, while the remaining techniques are also applicable to highthroughput approaches, and are cell-based. The application of these assays as screening platforms resulted in the discovery and validation of STAT3 dimerization inhibitors JSI-124 [5], bendamustine [6], piperlongumine [8] and static [10]. Moreover, the assays were also utilized as tools to support the discovery of other STAT3 dimerization 
inhibitors such as STX-0119 [9], STA-21 [11] and LLL-12 [12] which were all initially identified by in-silico highthroughput screening, and additionally applied to LY5 [13], shikonin derivatives [14], "Compound 9" [15], HJC1-30 [24] and HJC0123 [16] and FLLL32 [17] (Figure 1) which were designed based on previously published chemical structures.

An understanding of the pathway for STAT3 activation and the individual roles and functions of each STAT3 domain allows the targeting and subsequent attenuation of STAT3 activity in a specific and selective manner. STAT3 consists of six domains with different functions in the signal transduction pathway. The domain organization of the protein from the $\mathrm{N}$ - to $\mathrm{C}$-terminus is as follows: the N-terminal domain (ND) which mediates the tetramerization of two STAT3 dimers when binding to the promoters of target genes [25, 26]; the coiled-coil domain responsible for interacting with other cytoplasmic proteins [27]; the DNA-binding domain (DBD) through which STAT3 binds to the promoter sequences of genes [28]; the linker domain which lies between the DNAbinding and Src homology 2 (SH2) domains; the $\mathrm{SH} 2$ domain which plays a role in dimer formation with another phosphorylated STAT3 monomer (via phosphotyrosine residue(s), (pY) in the transcriptional activation domain) for initial binding of STAT3 to DNA [29, 30]; and the transcriptional activation domain (TAD) at the C-terminus which includes the pY site(s) for facilitating STAT3 dimerization and also is involved in the interactions with other nucleoplasmic proteins for the activation of transcription [31].

Although one STAT3 dimerization inhibitor (C1889) has advanced to early-phase clinical studies, it did not progress beyond this point [32], suggesting that preventing STAT3 dimerization through targeting the $\mathrm{SH} 2$ domain or TAD might be an intractable approach. Therefore, we and others have focused on inhibiting STAT3 DNA-binding through targeting the DBD. The small-molecule STAT3 DBD inhibitor (inS3-54) was reported in the literature in 2014, using an EMSA-based assay to determine inhibition of DNA-binding [33]. Other small-molecule STAT3 DBD inhibitors reported subsequently include additional inS354 analogues [34], and niclosamide which was validated using ELISA [35] (Figure 2). Of the two approaches used in these studies, only ELISA is applicable to highthroughput screening of compounds. Therefore, the development of a new orthogonal assay for discovering STAT3 DBD inhibitors would be desirable. Herein, we present an optimized high-throughput applicable FP assay for monitoring the STAT3:DNA association, referred to as
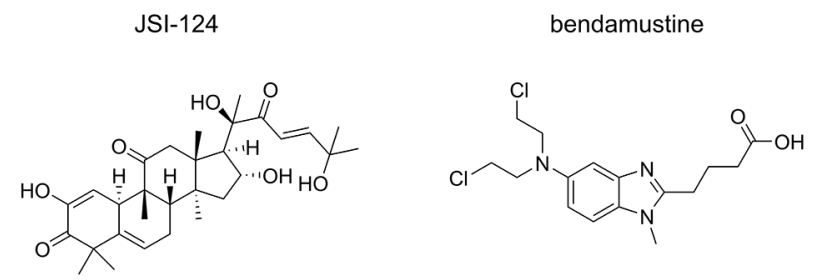

methanethiosulfonate derivative
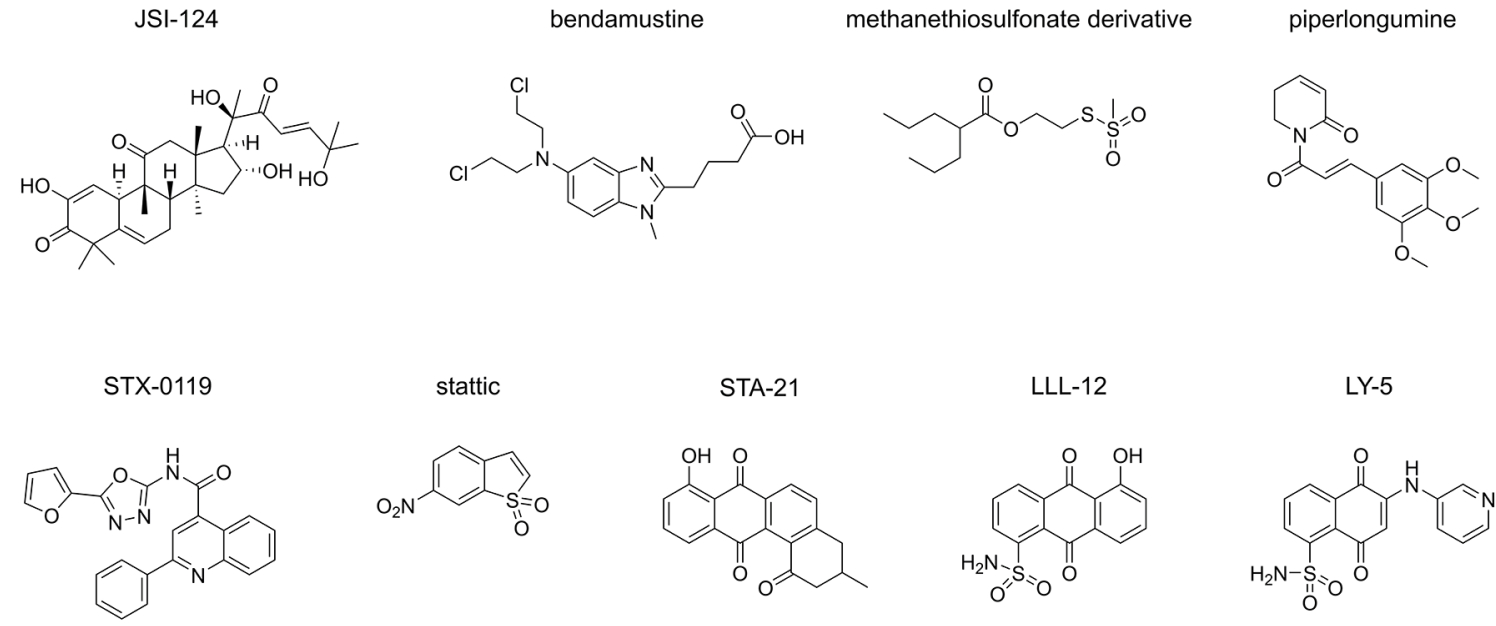

Compound 9

shikonin

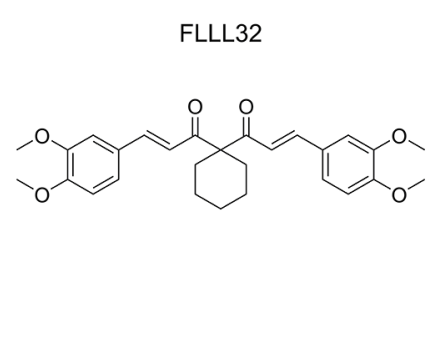

LLL-12

LY-5
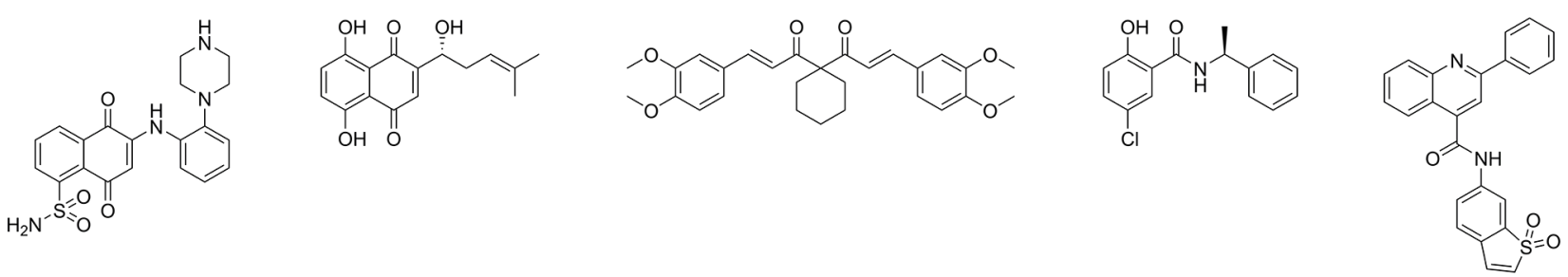

Figure 1: STAT3 dimerization inhibitors. Published STAT3 dimerization inhibitors validated by at least one of the following assays: FP assay, AlphaScreen ${ }^{\mathrm{TM}}$ assay, cytoblot assay, FRET assay, SPR assay and ELISA. 
the STAT3 ${ }^{127-688}$ :DNA FP assay. In brief, this assay uses a soluble STAT3 ${ }^{127-688}$ protein and a Bodipy-DNA conjugate as the fluorescent probe: the latter can be displaced by competitor ligands introduced during the experiment. The protocol is simple to implement compared to EMSA and ELISA, and there are no immobilised assay components, no addition of antibodies is required, and no washing procedures are involved, all of which impact on the time, cost and reliability of the assay.

\section{RESULTS}

\section{Optimized preparations: STAT3 ${ }^{127-688}$ target protein, and the Bodipy-DNA conjugate}

To prepare the STAT3 ${ }^{127-688}$ protein, an E. coli Rosetta strain was transformed with a recombinant pET-32a(+) plasmid containing the required STAT3 sequence (encoding residues 127 to 688) lacking the ND and TAD. The expressed crude protein was isolated and stored at $-20^{\circ} \mathrm{C}$ as pellets from ammonium sulphate precipitation. The crude protein was purified using ionexchange chromatography and the purified STAT3 ${ }^{127-688}$ was stored in the elution buffer $(\sim 200 \mathrm{mM} \mathrm{NaCl}, 1 \mathrm{mM}$ dithiothreitol (DTT), $25 \mathrm{mM}$ Tris $\mathrm{pH}$ 8.5). The conditions utilized in the subsequent STAT3 ${ }^{127-688}$ :DNA FP assays require the lowering of the salt and DTT concentrations by diafiltration using a $50 \mathrm{kDa}$ concentrator to a final $\mathrm{NaCl}$ concentration $<200 \mu \mathrm{M}$. The purified protein was examined by SDS-PAGE and found to be composed of a single component with a molecular weight consistent with that expected for the construct (Supplementary Figure 1A). An additional centrifugation step using a 300 $\mathrm{kDa}$ centrifugal filter removed misfolded, unfolded or aggregated STAT3 ${ }^{127-688}$ that may impact on DNA binding [36], and provided a protein that gave more consistent FP responses (Figure 3).

The Bodipy-DNA conjugate was purchased in the form of two complementary single-stranded DNA (ssDNA) sequences that were annealed in a salt- and DTT-free buffer (25 mM Tris pH 8.5). Bodipy 650/665 was selected as the fluorophore due to its relative insensitivity to $\mathrm{pH}$ changes and long absorption and emission wavelengths, which reduces the potential for fluorescence interference derived from aromatic smallmolecule inhibitors of the STAT3:DNA association.

\section{Optimization assays: $20 \mathrm{nM}$ Bodipy-DNA conjugate and $480 \mathrm{nM}$ STAT3 ${ }^{127-688}$}

The optimal working concentration of the BodipyDNA conjugate was determined in four separate FP experiments using either $1 \mathrm{nM}, 10 \mathrm{nM}, 20 \mathrm{nM}$, or $40 \mathrm{nM}$ of the probe for titration against increasing STAT3 ${ }^{127-688}$ concentrations. When the data were fitted to a one site saturation binding model, a good fit was observed for the $20 \mathrm{nM}$ and $40 \mathrm{nM}$ Bodipy-DNA concentrations, but not for $1 \mathrm{nM}$ and $10 \mathrm{nM}$ concentrations (Figure 4). Accordingly, a $20 \mathrm{nM}$ Bodipy-DNA concentration and a STAT3 ${ }^{127-688}$ concentration of $480 \mathrm{nM}$ (which gave $80 \%$ of the maximum FP response [37]) were selected for use in subsequent competition assays.

\section{Competition experiments with unlabelled DNA sequences}

To assess the displacement of the Bodipy-DNA conjugate, a 12-mer (12 base pair) unlabelled non-

\section{inS3-54}<smiles>O=C(O)c1ccc(N2C(=O)/C(=C/c3ccc([N+](=O)[O-])cc3)C=C2c2ccccc2)cc1</smiles>

inS3-54A18<smiles>O=C1/C(=C/c2ccc(Cl)cc2)C=C(c2ccccc2)N1c1ccc(O)cc1</smiles>

niclosamide<smiles>O=C(Nc1ccc([N+](=O)[O-])cc1Cl)c1cc(Cl)ccc1O</smiles>

Figure 2: STAT3 DBD inhibitors. Published STAT3 DBD inhibitors validated by at least one of the following assays: EMSA and ELISA. 
consensus DNA sequence or a 12-mer unlabelled consensus DNA sequence (identical to that of the BodipyDNA conjugate) were applied as competitive inhibitors to displace the 12-mer Bodipy-DNA conjugate from STAT3 ${ }^{127-688}$. The half-maximal inhibitory concentration $\left(\mathrm{IC}_{50}\right)$ of the consensus DNA was determined as $0.30 \pm$

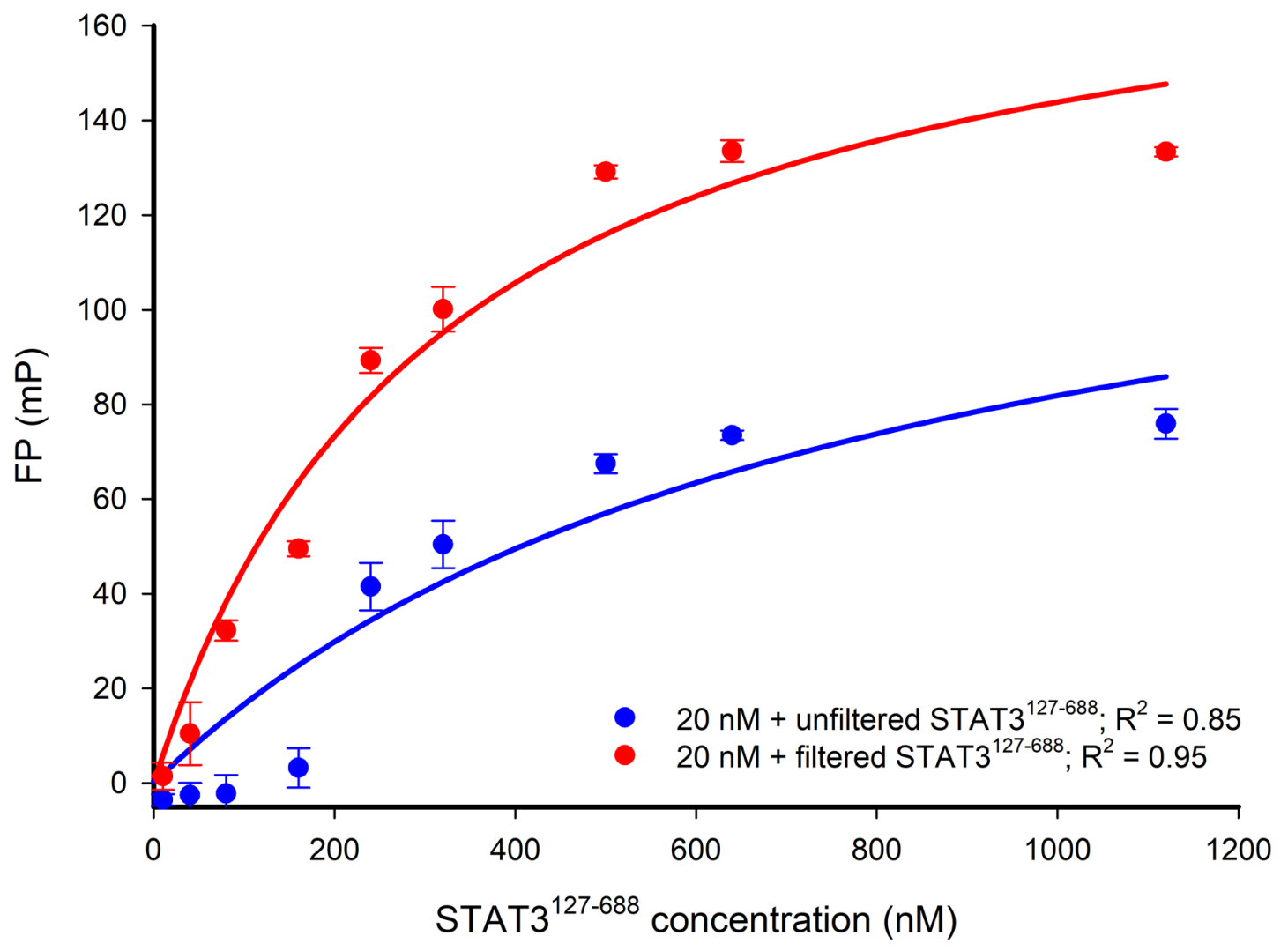

Figure 3: FP versus STAT3 ${ }^{127-688}$ concentration-response curves for 20 nM Bodipy-DNA conjugate applied with either filtered or unfiltered STAT3 ${ }^{127-688}$. The response curve obtained by using unfiltered STAT3 ${ }^{127-688}$ shows that low FP signals are detected when applying STAT3 ${ }^{127-688}$ concentrations lower than $200 \mathrm{nM}$. The filtration of STAT3 ${ }^{127-688}$ (300 kDa molecular weight cut-off filter) gave more consistent FP signal responses below $200 \mathrm{nM}$ STAT3 ${ }^{127-688}$ and an improved maximum FP signal. The $\mathrm{NaCl}$ concentration in each experiment was $<200 \mu \mathrm{M}$. $\mathrm{R}^{2}$ represents the coefficient of determination.
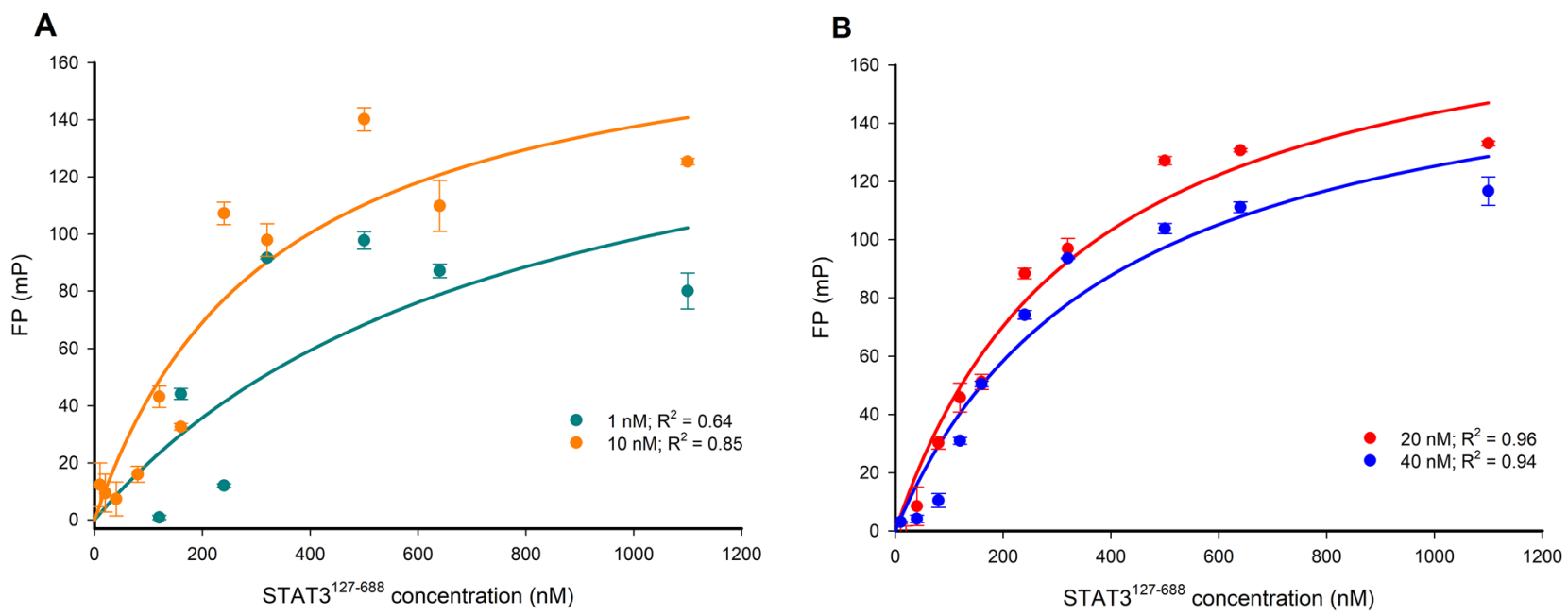

Figure 4: FP versus STAT3 ${ }^{127-688}$ concentration-response curves for varying concentrations of the Bodipy-DNA conjugate. (A) 1 and $10 \mathrm{nM}$ Bodipy-DNA conjugate; (B) 20 and $40 \mathrm{nM}$ Bodipy-DNA conjugate. $20 \mathrm{nM}$ was selected as the optimal Bodipy-DNA concentration. 
$0.20 \mu \mathrm{M}$, while the non-consensus DNA gave an $\mathrm{IC}_{50}$ of $2.3 \pm 0.66 \mu \mathrm{M}$ after $24 \mathrm{hr}$ (Figure 5). Although the nonconsensus DNA was not expected to bind to STAT3 ${ }^{127-}$ ${ }^{688}$, the result shows that there was an association but with a weaker binding affinity. This could be explained by non-specific binding due to electrostatic interactions between the DNA backbone and STAT3. This is consistent with previous observations that transcription factors can interact non-specifically with non-consensus sequences with lower binding affinities $[38,39]$.

\section{The STAT3:DNA association is time-dependent}

Before introducing small-molecule inhibitors into the assay, it is essential to understand the kinetics of the interaction between STAT3 $3^{127-688}$ and the Bodipy-DNA conjugate. Therefore, the equilibrium association of STAT3 $^{127-688}(480 \mathrm{nM})$ and Bodipy-DNA (20 nM) was monitored after various incubation times. Our results show that after 14 hours, an equilibrium was reached that was stable for at least $48 \mathrm{hr}$ (Figure 6). The length of time to reach equilibrium indicates slow binding kinetics between the two binding partners.

\section{Reliability and signal-to-noise: $Z^{\prime}$ values and S:N ratios}

The suitability of the STAT3 ${ }^{127-688}$ :DNA FP assay for high-throughput screening was assessed by measuring the $Z$ ' value (values $1>Z$ ' $\geq 0.5$ indicate a reliable assay $[40])$ and the signal-to-noise $(\mathrm{S}: \mathrm{N})$ ratio were determined at various incubation times (Table 1). Caution is required with interpreting the values of $\mathrm{Z}$ ' and $\mathrm{S}: \mathrm{N}$ ratios after short incubation times ( 0 and $1 \mathrm{hr}$ ) as equilibrium is achieved after $\geq 14 \mathrm{hr}$. However, values determined after $14 \mathrm{hr}$ are considered to be sufficiently robust to monitor competition between the Bodipy-DNA conjugate and inhibitors for the association with STAT3 ${ }^{127-688}$.

\section{Validation of the assay: inS3-54, inS3-54A18 and niclosamide are inhibitors of the STAT3:DNA association}

To understand the applicability of the STAT3 ${ }^{127-688}$ :DNA FP assay in compound screening campaigns, a series of pilot competition studies were conducted with known STAT3 inhibitors. The STAT3

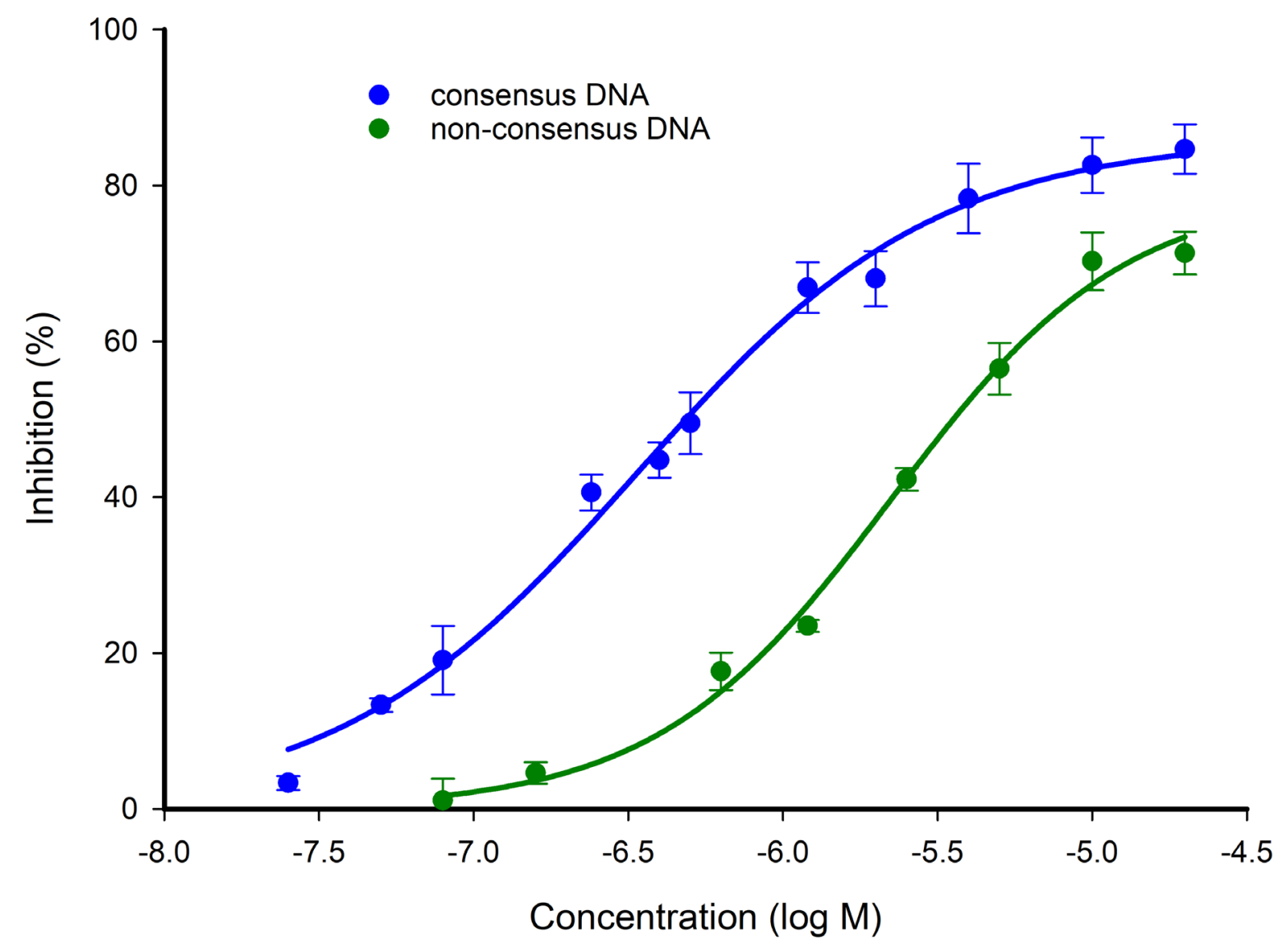

Figure 5: Competitive inhibition of Bodipy-DNA-STAT3 $3^{127-688}$ binding by consensus and non-consensus DNA sequences. The non-consensus DNA (self-complementary 5'-GTACCATGGTAC-3') bound to the protein with a lower affinity than the consensus DNA (5'-ATTTCCCGTAAA-3' and 5'-TTTACGGGAAAT-3'). Measurements were made after a $24 \mathrm{hr}$ incubation at $4^{\circ} \mathrm{C}$. 
Table 1: Calculated $Z$ ' values and $S: N$ ratios at various incubation times

\begin{tabular}{lcc}
\hline Time course & Z' values & S:N ratios \\
\hline $0 \mathrm{hr}$ & 0.53 & 9.16 \\
$1 \mathrm{hr}$ & 0.71 & 27.39 \\
$14 \mathrm{hr}$ & 0.63 & 20.06 \\
$24 \mathrm{hr}$ & 0.71 & 19.65 \\
$36 \mathrm{hr}$ & 0.72 & 19.16 \\
$48 \mathrm{hr}$ & 0.74 & 15.78 \\
\hline
\end{tabular}

DBD inhibitors, inS3-54, inS3-54A18 and niclosamide were tested along with STAT3 dimerization inhibitors, GpYLPQTV and HJC-1-30. GpYLPQTV is a peptide sequence derived from the gp130 subunit reported to bind to the STAT3 SH2 domain [18]. The activities of inS354 , inS3-54A18 and niclosamide were found to be doseand time-dependent (time-dependent data not shown) as previously described [33-35]. Estimated $\mathrm{IC}_{50}$ values determined for inS3-54, inS3-54A18 and niclosamide were $21.3 \pm 6.9 \mu \mathrm{M}, 126 \pm 39.7 \mu \mathrm{M}$ and $219 \pm 43.4 \mu \mathrm{M}$ after $24 \mathrm{hr}$ respectively (Figure 7). On the other hand, GpYLPQTV and HJC-1-30 were poor inhibitors of the
STAT3:DNA association achieving only $50 \%$ and $70 \%$ inhibition respectively at $400 \mu \mathrm{M}$ after incubation for 24 hr (Figure 7).

\section{The $\mathrm{IC}_{50}$ determination of the STAT3 ${ }^{127-688}$ :DNA FP assay is comparable to the protein electrophoretic mobility shift assay (PEMSA)}

In order to confirm the binding behavior of selected ligands to the STAT3 ${ }^{127-688}$ construct, we expressed and purified a recombinant yellow fluorescent protein YFPSTAT3 ${ }^{127-688}$ fusion protein in the same manner as that for

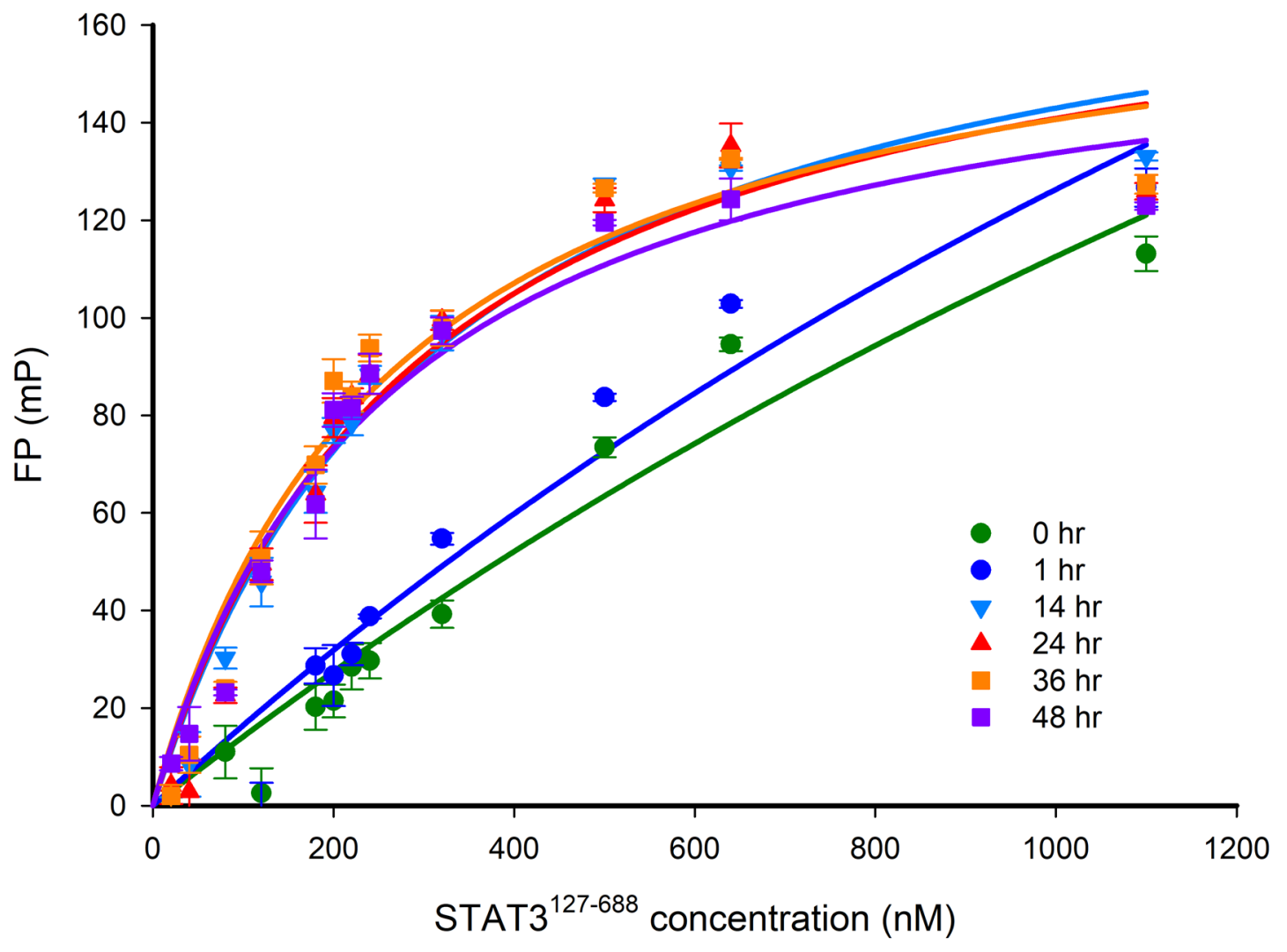

Figure 6: FP versus STAT3 ${ }^{127-688}$ concentration-response curves after different incubation times. The FP signal was measured after incubation of $20 \mathrm{nM}$ Bodipy-DNA conjugate and varying concentrations of STAT3 ${ }^{127-688}$ for between 0 and 48 hr. The response curves show that the STAT3:DNA association reached equilibrium after $14 \mathrm{hr}$. A STAT3 ${ }^{127-688}$ concentration of $480 \mathrm{nM}$ gave $80 \%$ of the maximum FP response, which was selected for use in competition experiments. 
STAT3 ${ }^{127-688}$ which were adapted from the method used for a STAT3 ${ }^{127-722}$ construct as previously described [41] (see Supplementary Materials and Methods). The purity of YFP-STAT3 ${ }^{127-688}$ is shown in Supplementary Figure 1B. A protein electrophoretic mobility shift assay (PEMSA) [41], that is under further development in our labs using YFP-STAT3 ${ }^{127-688}$ and the 12-mer unlabelled consensus DNA for STAT3, was carried out to give estimated $\mathrm{IC}_{50}$ values for the inhibitors. The values for inS3-54 and inS3$54 \mathrm{~A} 18$ were $\sim 26 \mu \mathrm{M}$ and $\sim 165 \mu \mathrm{M}$, respectively, after 24 hr incubation at $4^{\circ} \mathrm{C}$ (Supplementary Figures 2 and 3), which are comparable to those determined by the FP assay $(21.3 \pm 6.9 \mu \mathrm{M}, 126 \pm 39.7 \mu \mathrm{M}$ respectively).

\section{DISCUSSION}

Since STAT3 is highly involved in oncogenic pathways, there has been significant research directed towards finding inhibitors that interact with STAT3 or its upstream effectors. Identification of compounds that inhibit STAT3 dimerization has been a major focus. C188-9 has progressed to early-stage clinical trials [32], although it did not advance beyond this point. Recently, there has been an increased focus on blocking the interaction between STAT3 and DNA; however, the lack of direct binding assays has hindered structure-based drug design efforts in this area.

Consequently, we have developed a fluorescence polarization method to quantify STAT3 DNA-binding using a double-stranded DNA-fluorophore conjugate that has the consensus sequence 5'-TTNCNNNAA-3' [42] tethered to Bodipy at the 5' end. The assay utilizes a STAT3 ${ }^{127-688}$ construct, which lacks the Tyr705containing transactivation domain (TAD) that is involved in phosphorylation-mediated dimerization of STAT3

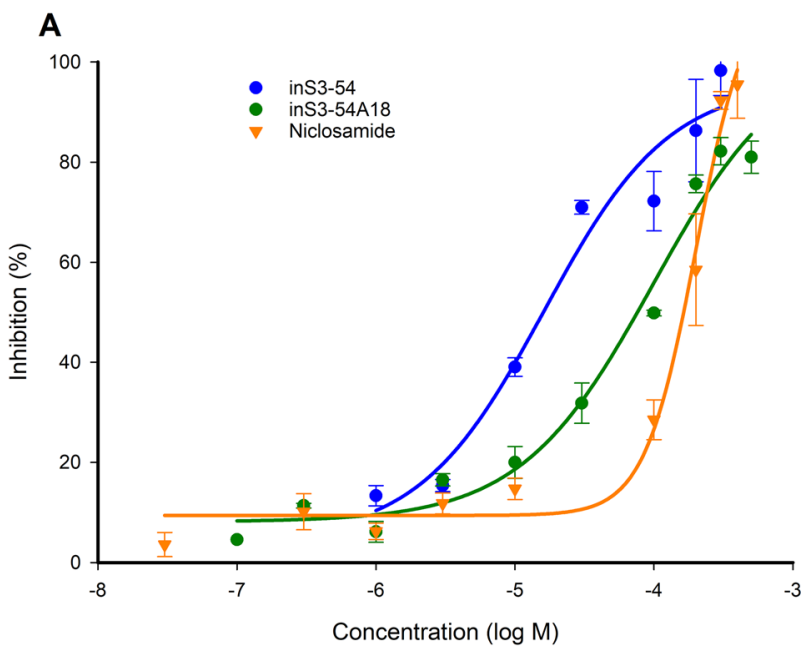

and omits the N-terminal domain (ND) that is involved in tetramerization. Thus, the assay should be useful for identifying compounds that inhibit the STAT3:DNA association via binding to non-TAD/ND sites. The assay is a cell-free alternative to the EMSA and ELISA that have been applied previously in STAT3 DNA-binding inhibitor screening studies $[33,35]$. This means that effects on upstream proteins or off-target effects on STAT3 cellular concentrations can be ruled out. The use of $\left[{ }^{32} \mathrm{P}\right]$ endlabelled DNA, as is the case with EMSA, is avoided, and the use of recombinant STAT3 protein removes the need for cell nuclear extracts as a source of STAT3 protein which was utilized in the cell-based ELISA protocol as described by Furtek et al. [35].

The FP assay has some advantages in terms of throughput compared to ELISA and EMSA-based methods. Unlike EMSA, the FP-based experiments can be carried out in microtiter plates and are amenable to parallelization and automation. The assay is homogeneous unlike the ELISA method that requires washing steps and the measurement is direct, in contrast to ELISA that requires antibodymediated amplification of the readout. Additionally, the STAT3 ${ }^{127-688}$ :DNA FP assay described here, and the related STAT3-phosphopeptide FP technique utilizing FITC-GpYLPQTV as an SH2 domain-interactive probe (adapted from the method of Schust \& Berg) [18] can be performed concurrently in two halves of the same 96-well black microtiter plate using suitable optical modules for each labelled probe (data not shown). Such an approach allows comparisons of the displacement of DNA and phosphopeptide fluorescent probes to be determined using the same protein construct, potentially allowing DBD and STAT3 dimerization inhibitors to be distinguished.

The applicability of the STAT3 ${ }^{127-688}$ :DNA FP assay for high-throughput screening was validated using previously

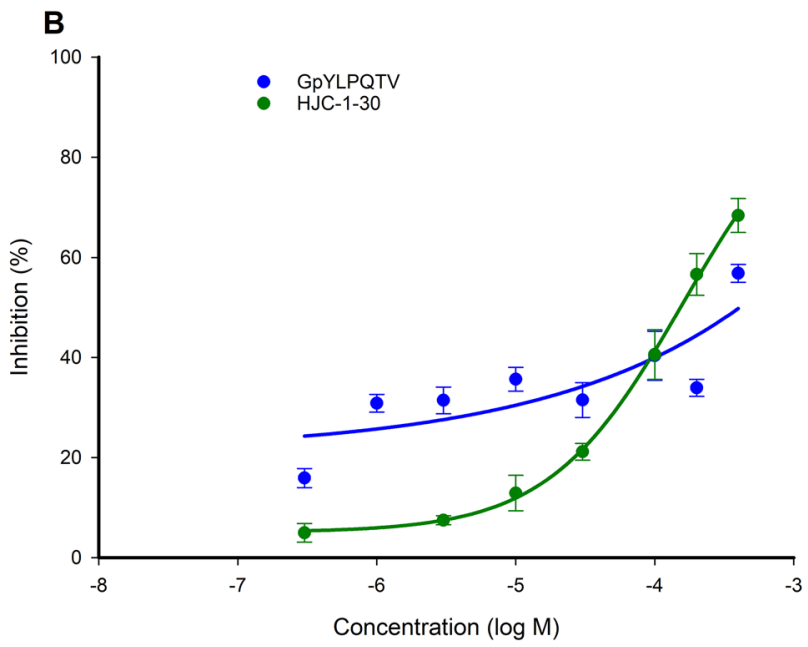

Figure 7: Validation of the STAT3 ${ }^{127-688}$ :DNA FP competition assay using published STAT3 inhibitors. Percentage inhibition of Bodipy-DNA binding versus inhibitor concentration response curves for (A) STAT3 DBD inhibitors inS3-54, inS3-54A18 and niclosamide and (B) STAT3 dimerization inhibitors GpYLPQTV and HJC-1-30. Measurements were made after a $24 \mathrm{hr}$ incubation at $4{ }^{\circ} \mathrm{C}$. 
described STAT3 DBD and dimerization inhibitors (Figures 5 and 7). $\mathrm{IC}_{50}$ values determined after $24 \mathrm{hr}$ were: inS3-54, 21.3 $\pm 6.9 \mu \mathrm{M}$; inS3-54A18, $126 \pm 39.7 \mu \mathrm{M}$; and niclosamide, $219 \pm 43.4 \mu \mathrm{M}$ (Figure 5). The binding affinities of inS354 and S3-54A18 were further validated using the YFPSTAT3 ${ }^{127-688}$ :DNA PEMSA that is under further development in our laboratories (Supplementary Figures 2 and 3). These values are not entirely consistent with those reported using cell-based assays using either the STAT3-dependent luciferase reporter assay $\left(\mathrm{IC}_{50} \sim 14 \mu \mathrm{M}\right.$ for inS3-54 and $\sim 11$ $\mu \mathrm{M}$ for inS3-54A18) or the ELISA-based method $\left(\mathrm{IC}_{50} \sim 0.2\right.$ $\mu \mathrm{M}$ for niclosamide) $[32,33,35]$. This raises the possibility that inS3-54 and inS3-54A18 may have additional activities in cells that contribute to the inhibition of STAT3-dependent luciferase reporter activity that warrant further investigation. A similar rationale may apply to niclosamide-treated HeLa cells from which nuclear extracts are taken for use in the ELISA-based detection of STAT3 activity [35].

The data support the use of the assay for characterizing both small molecule- and DNA-based competitors; the latter may be useful for quantifying the binding affinity of various consensus STAT3 binding sequences (Figures 5 and 7). The reliability of this FP assay was verified by the calculation of Z' values and S:N ratios at time points up to $48 \mathrm{hr}$ (Table 1). Refinement of the time-dependent activities of STAT3 inhibitors is possible; however, the slow association kinetics of STAT3 ${ }^{127-688}$ and the Bodipy-DNA probe would need to be considered when carrying out such experiments (Figure 6).

Taken together, our STAT3 ${ }^{127-688}$ :DNA FP assay is a useful addition to the available assays for discovering STAT3 DBD inhibitors, because this assay is: 1) applicable to high-throughput screenings using both small moleculebased and nucleic acid-based libraries in a multi-well plate format, 2) suitable for the validation of the inhibitory effect on the STAT3:DNA binding for inhibitors, 3) able to determine dose- and time-dependent activities of STAT3 DBD inhibitors, 4) simple to conduct in comparison with the ELISA and EMSA methods, and 5) can be performed in parallel with the STAT3-phosphopeptide FP assay for discovering dimerization inhibitors on the same plate. In principle the assay methodology could be applied to other members of the STAT family of transcription factors including STAT1 and STAT5; both proteins have been cloned and expressed, and STAT1 has been co-crystallized with DNA facilitating the design of equivalent protein constructs for FP assays [43-45].

\section{MATERIALS AND METHODS}

\section{Preparation of inS3-54, inS3-54A18, niclosamide, GpYLPQTV and HJC-1-30}

Both inS3-54 and inS3-54A18 were synthesized using methods adapted from those described by Huang et al. [34]. The chemical structures of inS3-54 and inS3-54A18 were confirmed by ${ }^{1} \mathrm{H} \mathrm{NMR},{ }^{13} \mathrm{C} \mathrm{NMR}$ and LC/MS and analytical purities of $>95 \%$ were recorded $\left({ }^{1} \mathrm{H}\right.$ NMR and LC/MS). Niclosamide and GpYLPQTV were purchased in a powder form from Sigma-Aldrich and Generon, respectively. HJC-1-30 was provided by Dr Charlie Nichols. The inhibitors were dissolved in 100\% DMSO to form stock solutions for the STAT3 ${ }^{127-688}$ :DNA FP assay.

\section{Oligonucleotides}

All oligonucleotide sequences were purchased from Eurofins as freeze-dried powders. The sequence of the unlabelled non-consensus DNA was selfcomplementary 5'-GTACCATGGTAC-3'. The sequences of the Bodipy-DNA conjugate 5'-ATTTCCCGTAAA-3' and 5'-TTTACGGGAAAT-3' and the unlabelled consensus DNA are identical. The fluorophore, Bodipy $650 / 665$, was chemically linked to the 5 ' end of 5'-ATTTCCCGTAAA-3'. The Bodipy-DNA conjugate sequence was designed based upon M67 core sequence 5'-TTCCCGTAA-3'[46], while the unlabelled nonconsensus DNA was designed to minimise similarity with the STAT3 consensus sequence 5'-TTNCNNNAA-3' [42].

\section{DNA annealing}

The oligonucleotides were dissolved in the annealing buffer ( $25 \mathrm{mM}$ Tris $\mathrm{pH} 8.5$ ). An equal volume and concentration of each ssDNA was mixed in an Eppendorf tube that was then incubated at $95^{\circ} \mathrm{C}$ for $3 \mathrm{~min}$, followed by cooling to room temperature overnight (in a $1 \mathrm{~kg}$ heatblock). This double-stranded DNA was then stored at $-20^{\circ} \mathrm{C}$. The Bodipy-DNA conjugate was prepared in the dark to prevent photoquenching of the fluorophore.

\section{STAT3 $^{127-688}$ expression and purification}

STAT3 ${ }^{127-688}$ expression was conducted as previously described [41]. One day before the STAT3 ${ }^{127-688}$ :DNA FP assay was performed, the ammonium sulphate-precipitated crude protein pellet was re-suspended using a solution of $1 \mathrm{mM}$ DTT $25 \mathrm{mM}$ Tris $\mathrm{pH} 8.5$ and purified using HiTrap QFF columns (GE Healthcare) coupled to a fast protein column chromatography (FPLC) instrument (NGCTM Chromatography Systems, Bio-Rad). The elution of the STAT3 ${ }^{127-688}$ protein was conducted using a solution of $1 \mathrm{mM}$ DTT and $25 \mathrm{mM}$ Tris pH 8.5, and a gradient of increasing $\mathrm{NaCl}$ concentration. The purified STAT3 ${ }^{127-688}$ usually eluted at $200 \mathrm{mM} \mathrm{NaCl}$ and was collected in a 96-well block. The purified STAT3 ${ }^{127-688}$ was stored in the eluent at $4^{\circ} \mathrm{C}$. The purity of STAT3 ${ }^{127-688}$ was determined using SDS-PAGE.

\section{Preparation of the STAT3 ${ }^{127-688}$ sample for the STAT3 $^{127-688}$ :DNA FP assay}

On the day the STAT3 ${ }^{127-688}$ :DNA FP assay was performed, the STAT3 ${ }^{127-688}$ protein sample was 
prepared immediately for use, usually at $0.4 \mathrm{mg} / \mathrm{ml}$. Buffer exchange was performed at least three times by diafiltration conducted using a $50 \mathrm{kDa}$ centrifugal concentrator (Sartorius). The residual STAT3 ${ }^{127-688}$ protein solution was diluted 10-fold with diafiltration buffer, 25 $\mathrm{mM}$ Tris $\mathrm{pH} 8.5$, upon completion of each diafiltration step. Aggregated STAT3 ${ }^{127-688}$ was removed using a benchtop centrifuge if aggregation was observed on visual inspection of the samples. Finally, invisible STAT3 ${ }^{127-688}$ aggregate was removed from the sample by centrifugation through a $300 \mathrm{kDa}$ centrifugal filter (Sartorius) at $\sim 800 \mathrm{~g}$.

\section{Conduct of the STAT3 ${ }^{127-688}$ :DNA FP assay}

The FP assay was conducted in 96-well Chimney Well black microtiter plates (Greiner Bio-One). The final concentration of FP buffer was composed of $5 \%$ glycerol, $20 \mathrm{mM}$ Tris $\mathrm{pH} 8.5,1 \mathrm{mM}$ EDTA, $0.01 \mathrm{mg} / \mathrm{ml}$ bovine serum albumin, 4\% DMSO, with or without $480 \mathrm{nM}$ purified STAT3 ${ }^{127-688}, 20$ nM Bodipy-DNA conjugate, with or without a variable concentration of inhibitor, to make a total volume of $100 \mu \mathrm{l}$ per well. After the addition of all assay components, the plates were incubated and gently agitated for the first hour at room temperature, followed by further incubation at $4^{\circ} \mathrm{C}$. During incubation, the plates were covered with black lids to protect the samples from light and to reduce evaporation of the FP buffer solution. The plates were read at various incubation times using an FP plate reader (PHERAstar, BMG Labtech) with an FP 590-675-675 optical module (BMG Labtech).

\section{Calculations of $Z$ ' value and $S: N$ ratio}

The Z' values were calculated using the equation $\mathrm{Z}$ ' = 1 - $\left(3 \mathrm{SD}_{\text {bound }}+3 \mathrm{SD}_{\text {free }}\right) /\left(\right.$ mean of $\mathrm{mP}_{\text {bound }}-$ mean of $\left.\mathrm{mP}_{\text {free }}\right)$, where the $\mathrm{SD}$ is the standard deviation of the measurements and the $\mathrm{mP}$ is the measured fluorescence polarization [40]. The $\mathrm{mP}$ was automatically calculated by the FP plate reader using the equation $\mathrm{mP}=\left(\mathrm{I}_{\|}-\mathrm{I}_{\perp}\right) /\left(\mathrm{I}_{\|}+\mathrm{I}_{\perp}\right)$ where $\mathrm{I}_{\|}$and $\mathrm{I}_{\perp}$ are the fluorescence polarization intensities of the Bodipy-DNA conjugate with polarizations parallel and perpendicular to the incident light, respectively. The bound state was determined by incubating $20 \mathrm{nM}$ Bodipy-DNA conjugate with 480 nM STAT3 ${ }^{127-688}$ protein, whereas for the free state, the same mixture was incubated with an additional $10 \mu \mathrm{M}$ of unlabelled consensus DNA as a competitor. The equation $\mathrm{S}: \mathrm{N}=\left(\right.$ mean of $\mathrm{mP}_{\text {bound }}-$ mean of $\left.\mathrm{mP}_{\text {free }}\right) /\left(\mathrm{SD}_{\text {bound }}{ }^{2}+\mathrm{SD}_{\text {free }}{ }^{2}\right)^{0.5}$ was utilized to determine the signal-to-noise ratios [47].

\section{Analytical methodology}

Each assay condition was evaluated with at least three repeats. Binding curves were fitted using SigmaPlot 13 using the 'one site saturation' and 'sigmoidal doseresponse (variable slope)' non-linear regression curvefitting functions. The 'one site saturation' model uses the equation $\mathrm{y}=\left(\mathrm{B}_{\max } \mathrm{x}\right) /\left(\mathrm{K}_{\mathrm{D}}+\mathrm{x}\right)$, where $\mathrm{B}_{\max }$ is the maximal
FP response and $K_{D}$ is the dissociation constant. The 'sigmoidal dose-response (variable slope)' utilizes the equation $\mathrm{y}=\min +(\max -\min ) /\left(1+10^{\mathrm{b}(\log I C 50-\mathrm{x})}\right)$, where max and min represents the maximum and minimum response plateaus respectively, and $\mathrm{b}$ is the Hill slope for the binding event. The error bars shown in the graphs are SDs from the mean values of the replicates.

\section{Abbreviations}

12-mer, 12 base pair; DBD, DNA-binding domain; DTT, dithiothreitol; ELISA, enzyme-linked immunosorbent assay; EMSA, electrophoretic mobility shift assay; FP, fluorescence polarization; FRET, fluorescence resonance energy transfer; ND, N-terminal domain; PEMSA, protein electrophoretic mobility shift assay; pY, phosphorylated tyrosine; SD, standard deviation; SH2, SRC homology 2; S:N, signal-to-noise ratio; SPR, surface plasmon resonance; STAT3, signal transducer and activator of transcription 3; STAT3 $3^{127-688}$, a truncated STAT3 with residues 127-688; TAD, transcriptional activation domain.

\section{Author contributions}

Po-Chang Shih conducted the experiments except for the preparations of recombinant STAT3 ${ }^{127-688}$ pET$32 \mathrm{a}(+)$ and YFP-STAT3 ${ }^{127-688}$ pET-32a $(+)$ plasmids. Yiwen Yang constructed the YFP-STAT3 ${ }^{127-688}$ pET-32a $(+)$ plasmid and advised on the conduct of the PEMSA. Gary Parkinson provided consumables for the establishment and optimization of the STAT3 ${ }^{127-688}$ :DNA FP assay and the PEMSA with YFP-STAT3 $3^{127-688}$ and the 12-mer DNA. Andrew Wilderspin provided the recombinant STAT3 ${ }^{127-688}$ pET-32a $(+)$ plasmid, FP-related instruments for carrying out this STAT3 ${ }^{127-688}$ :DNA FP assay and PEMSA-related knowledge and instruments. Geoffrey Wells provided advice and resources for chemical synthesis. PS, GP, AW and GW provided critical advice on the design of the experiments and contributed to writing and reviewing the manuscript.

\section{ACKNOWLEDGMENTS}

We thank Dr. Charlie Nichols for providing the HJC1-30 inhibitor and adapting the STAT3-phosphopeptide FP assay based on the method of Schust and Berg [18].

\section{CONFLICTS OF INTEREST}

The authors declare no conflicts of interest.

\section{FUNDING}

We would like to thank the Worldwide Cancer Research (Grant Ref 13-0133, GP and AW) and UCL 
School of Pharmacy for financial support (PS, GP, AW, and GW). HRMS were obtained from the EPSRC UK National Mass Spectrometry Facility (NMSF) at Swansea University.

\section{REFERENCES}

1. Haura EB. SRC and STAT pathways. Journal of Thoracic Oncology. 2006; 1:403-5. https://doi.org/10.1016/S1556-0864(15)31601-4.

2. Harrison DA. The JAK/STAT pathway. Cold Spring Harb Perspect Biol. 2012; 4:a011205. https://doi.org/10.1101/cshperspect.a011205.

3. Fagard R, Metelev V, Souissi I, Baran-Marszak F. STAT3 inhibitors for cancer therapy: have all roads been explored? JAKSTAT. 2013; 2:e22882. https://doi.org/10.4161/jkst.22882.

4. Siveen KS, Sikka S, Surana R, Dai X, Zhang J, Kumar AP, Tan BK, Sethi G, Bishayee A. Targeting the STAT3 signaling pathway in cancer: role of synthetic and natural inhibitors. Biochim Biophys Acta. 2014; 1845:136-54. https://doi.org/10.1016/j.bbcan.2013.12.005.

5. Blaskovich MA, Sun J, Cantor A, Turkson J, Jove R, Sebti SM. Discovery of JSI-124 (cucurbitacin I), a selective Janus kinase/signal transducer and activator of transcription 3 signaling pathway inhibitor with potent antitumor activity against human and murine cancer cells in mice. Cancer Res. 2003; 63:1270-9.

6. Iwamoto K, Uehara Y, Inoue Y, Taguchi K, Muraoka D, Ogo N, Matsuno K, Asai A. Inhibition of STAT3 by anticancer drug bendamustine. PLoS One. 2017; 12:1-18. https://doi.org/10.1371/journal.pone.0170709.

7. Gabriele E, Ricci C, Meneghetti F, Ferri N, Asai A, Sparatore A. Methanethiosulfonate derivatives as ligands of the STAT3-SH2 domain. J Enzyme Inhib Med Chem. 2017; 32:337-44. https://doi.org/10.1080/14756366.2016.1252757.

8. Bharadwaj U, Eckols TK, Kolosov M, Kasembeli MM, Adam A, Torres D, Zhang X, Dobrolecki LE, Wei W, Lewis MT, Dave B, Chang JC, Landis MD, et al. Drugrepositioning screening identified piperlongumine as a direct STAT3 inhibitor with potent activity against breast cancer. Oncogene. 2015; 34:1341-53. https://doi.org/10.1038/onc.2014.72.

9. Matsuno K, Masuda Y, Uehara Y, Sato H, Muroya A, Takahashi O, Yokotagawa T, Furuya T, Okawara T, Otsuka M, Ogo N, Ashizawa T, Oshita C, et al. Identification of a new series of STAT3 inhibitors by virtual screening. ACS Med Chem Lett. 2010; 1:371-5. https://doi.org/10.1021/ml1000273.

10. Schust J, Sperl B, Hollis A, Mayer TU, Berg T. Stattic: A Small-Molecule Inhibitor of STAT3 Activation and Dimerization. Chem Biol. 2006; 13:1235-42. https://doi.org/10.1016/j.chembiol.2006.09.018.

11. Song H, Wang R, Wang S, Lin J. A low-molecularweight compound discovered through virtual database screening inhibits Stat3 function in breast cancer cells. Proc Natl Acad Sci U S A. 2005; 102:4700-5. https://doi.org/10.1073/pnas.0409894102.

12. Lin L, Hutzen B, Li PK, Ball S, Zuo M, DeAngelis S, Foust E, Sobo M, Friedman L, Bhasin D, Cen L, Li C, Lin J. A novel small molecule, LLL12, inhibits STAT3 phosphorylation and activities and exhibits potent growthsuppressive activity in human cancer cells. Neoplasia. 2010; 12:39-50. https://doi.org/10.1593/neo.91196.

13. Yu W, Xiao H, Lin J, Li C. Discovery of novel STAT3 small molecule inhibitors via in silico site-directed fragmentbased drug design. J Med Chem. 2013; 56:4402-12. https://doi.org/10.1021/jm400080c.

14. Qiu H, Zhu X, Luo Y, Lin H, Tang C, Qi J. Identification of new shikonin derivatives as antitumor agents targeting STAT3 SH2 domain. Sci Rep. 2017; 7:1-13. https://doi.org/10.1038/s41598-017-02671-7.

15. Yu W, Li C, Zhang W, Xia Y, Li S, Lin JY, Yu K, Liu M, Yang L, Luo J, Chen Y, Sun H, Kong L. Discovery of an orally selective inhibitor of signal transducer and activator of transcription 3 using advanced multiple ligand simultaneous docking. J Med Chem. 2017; 60:2718-31. https://doi.org/10.1021/acs.jmedchem.6b01489.

16. Chen H, Yang Z, Ding C, Chu L, Zhang Y, Terry K, Liu H, Shen Q, Zhou J. Fragment-based drug design and identification of HJC0123, a novel orally bioavailable STAT3 inhibitor for cancer therapy. Eur J Med Chem. 2013; 62:498-507. https://doi.org/10.1016/j.ejmech.2013.01.023.

17. Lin L, Deangelis S, Foust E, Fuchs J, Li C, Li PK, Schwartz EB, Lesinski GB, Benson D, Lü J, Hoyt D, Lin J. A novel small molecule inhibits STAT3 phosphorylation and DNA binding activity and exhibits potent growth suppressive activity in human cancer cells. Mol Cancer. 2010; 9:217. https://doi.org/10.1186/1476-4598-9-217.

18. Schust J, Berg T. A high-throughput fluorescence polarization assay for signal transducer and activator of transcription 3. Anal Biochem. 2004; 330:114-8. https://doi.org/10.1016/j.ab.2004.03.024.

19. Uehara Y, Mochizuki M, Matsuno $K$, Haino $T$, Asai A. Novel high-throughput screening system for identifying STAT3-SH2 antagonists. Biochem Biophys Res Commun. 2009; 380:627-31. https://doi.org/10.1016/j.bbrc.2009.01.137.

20. Kretzschmar AK, Dinger MC, Henze C, Brocke-Heidrich K, Horn F. Analysis of Stat3 (signal transducer and activator of transcription 3) dimerization by fluorescence resonance energy transfer in living cells. Biochem J. 2004; 377:28997. https://doi.org/10.1042/bj20030708.

21. Haan S, Hemmann U, Hassiepen U, Schaper F, SchneiderMergener J, Wollmer A, Heinrich PC, Grötzinger J. Characterization and binding specificity of the monomeric STAT3-SH2 domain. J Biol Chem. 1999; 274:1342-8. https://doi.org/10.1074/jbc.274.3.1342. 
22. Stockwell BR, Haggarty SJ, Schreiber SL. Highthroughput screening of small molecules in miniaturized mammalian cell-based assays involving posttranslational modifications. Chem Biol. 1999; 6:71-83. https://doi.org/10.1016/s1074-5521(99)80004-0.

23. Turkson J, Bowman T, Adnane J, Zhang YI, Djeu JY, Sekharam M, Frank DA, Holzman LB, Wu JIE, Sebti S, Jove R. Requirement for Ras/Rac1-mediated p38 and c-Jun $\mathrm{N}$-terminal kinase signaling in Stat 3 transcriptional activity induced by the Src oncoprotein. Mol Cell Biol. 1999; 19:7519-28. https://doi.org/10.1128/mcb.19.11.7519.

24. Zhou J, Chen H, Shen Q, inventors; University of Texas System, assignee. STAT3 inhibitor. US patent US20170152260A1. 2013 Jan 15.

25. John S, Vinkemeier U, Soldaini E, Darnell JE, Leonard WJ. The significance of tetramerization in promoter recruitment by Stat5. Mol Cell Biol. 1999; 19:1910-8. https://doi.org/10.1128/mcb.19.3.1910.

26. Vinkemeier U, Cohen SL, Moarefi I, Chait BT, Kuriyan J, Darnell JE. DNA binding of in vitro activated Stat1 alpha, Stat1 beta and truncated Stat1: interaction between NH2-terminal domains stabilizes binding of two dimers to tandem DNA sites. EMBO J. 1996; 15:5616-26.

27. Ma J, Zhang T, Novotny-Diermayr V, Tan AL, Cao X. A novel sequence in the coiled-coil domain of Stat3 essential for its nuclear translocation. J Biol Chem. 2003; 278:29252-60. https://doi.org/10.1074/jbc.m304196200.

28. Kamran MZ, Patil P, Gude RP. Role of STAT3 in cancer metastasis and translational advances. BioMed Research International. 2013; 2013:421821. https://doi.org/10.1155/2013/421821.

29. Darnell JE. STATs and gene regulation. Science. 1997; 277:1630-5. https://doi.org/10.1126/science.277.5332.1630.

30. Turkson J. STAT proteins as novel targets for cancer drug discovery. Expert Opin Ther Targets. 2004; 8:409-22. https://doi.org/10.1038/sj.onc.1204086.

31. Horvath CM. STAT proteins and transcriptional responses to extracellular signals. Trends Biochem Sci. 2000; 25:496502. https://doi.org/10.1016/s0968-0004(00)01624-8.

32. Bharadwaj U, Eckols TK, Xu X, Kasembeli MM, Adachi M, Song Y, Mo Q, Lai SY, David J. Small-molecule inhibition of STAT3 in radioresistant head and neck squamous cell carcinoma. Oncotarget. 2016; 7:26307-30. https://doi.org/10.18632/oncotarget.8368.

33. Huang W, Dong Z, Wang F, Peng H, Liu JY, Zhang JT. A small molecule compound targeting STAT3 DNA-binding domain inhibits cancer cell proliferation, migration, and invasion. ACS Chem Biol. 2014; 9:1188-96. https://doi.org/10.1021/cb500071v.

34. Huang W, Dong Z, Chen Y, Wang F, Wang CJ, Peng H, He Y, Hangoc G, Pollok K, Sandusky G, Fu X, Broxmeyer HE. Small-molecule inhibitors targeting the DNA-binding domain of STAT3 suppress tumor growth, metastasis and
STAT3 target gene expression in vivo. Oncogene. 2015; 35:783-92. https://doi.org/10.1038/onc.2015.215.

35. Furtek SL, Matheson CJ, Backos DS, Reigan P. Evaluation of quantitative assays for the identification of direct signal transducer and activator of transcription 3 (STAT3) inhibitors. Oncotarget. 2016; 7:77998-8008. https://doi.org/10.18632/oncotarget.12868.

36. Butturini E, Gotte G, Dell'Orco D, Chiavegato G, Marino V, Canetti D, Cozzolino F, Monti M, Pucci P, Mariotto S. Intermolecular disulfide bond influences unphosphorylated STAT3 dimerization and function. Biochem J. 2016; 473: 3205-19. https://doi.org/10.1042/bcj20160294.

37. Moerke NJ. Fluorescence polarization (FP) assays for monitoring peptide-protein or nucleic acid-protein binding. Current Protocols in Chemical Biology. 2009; 1:1-15. https://doi.org/10.1002/9780470559277.ch090102.

38. Badis G, Berger MF, Philippakis AA, Talukder S, Gehrke AR, Jaeger SA, Chan ET, Metzler G, Vedenko A, Chen X, Kuznetsov H, Wang CF, Coburn D, et al. Diversity and complexity in DNA recognition by transcription factors. Science. 2009; 324:1720-3. https://doi.org/10.1126/science.1162327.

39. Afek A, Schipper JL, Horton J, Gordân R, Lukatsky DB. Protein-DNA binding in the absence of specific base-pair recognition. Proc Natl Acad Sci U S A. 2014; 111:17140-5. https://doi.org/10.1073/pnas.1410569111.

40. Zhang JH. A simple statistical parameter for use in evaluation and validation of high throughput screening assays. J Biomol Screen. 1999; 4:67-73. https://doi.org/10.1177/108705719900400206.

41. Nkansah E, Shah R, Collie GW, Parkinson GN, Palmer J, Rahman KM, Bui TT, Drake AF, Husby J, Neidle S, Zinzalla G, Thurston DE, Wilderspin AF. Observation of unphosphorylated STAT3 core protein binding to target dsDNA by PEMSA and X-ray crystallography. FEBS Lett. 2013; 587:833-9. https://doi.org/10.1016/j.febslet.2013.01.065.

42. Horvath CM, Wen Z, Darnell JE. A STAT protein domain that determines DNA sequence recognition suggests a novel DNA-binding domain. Genes Dev. 1995; 9:984-94. https://doi.org/10.1101/gad.9.8.984.

43. Chen X, Vinkemeier U, Zhao Y, Jeruzalmi D, Darnell JE, Kuriyan J. Crystal structure of a tyrosine phosphorylated STAT-1 dimer bound to DNA. Cell. 1998; 93:827-39. https://doi.org/10.1016/s0092-8674(00)81443-9.

44. Neculai D, Neculai AM, Verrier S, Straub K, Klumpp K, Pfitzner E, Becker S. Structure of the unphosphorylated STAT5a dimer. J Biol Chem. 2005; 280:40782-7. https://doi.org/10.1074/jbc.m507682200.

45. Soldaini E, John S, Moro S, Bollenbacher J, Schindler U, Leonard WJ. DNA binding site selection of dimeric and tetrameric Stat5 proteins reveals a large repertoire of divergent tetrameric Stat5a 
binding sites. Mol Cell Biol. 2000; 20:389-401. https://doi.org/10.1128/mcb.20.1.389-401.2000.

46. Wagner BJ, Hayes TE, Hoban CJ, Cochran BH. The SIF binding element confers sis/PDGF inducibility onto the c-fos promoter. EMBO J. 1990; 9:4477-84.
47. Kim J. Development of a fluorescence polarization assay for the molecular chaperone Hsp90. J Biomol Screen. 2004; 9:375-81. https://doi.org/10.1177/1087057104265995. 